\title{
Transformaciones urbano-rurales: Hampaturi y Retamani ${ }^{1}$ Urban-rural transformations: Hampaturi and Retamani
}

\section{Carla Andrea Becerra Cardona ${ }^{2}$}

Resumen: hampaturi y Retamani son territorios que cada vez exponen una mayor articulación con la ciudad de La Paz. Así, estos territorios expresan un proceso de organización que no incluye a los límites administrativos municipales de los cuales son parte. Este artículo, basado en recorridos y en entrevistas, sugiere un análisis de la situación espacial a través de una descripción de las transformaciones que presenta el uso del suelo de ambos territorios. Se lo vincula al uso del suelo para la producción agrícola y cómo se vincula a los diferentes mercados de la ciudad de la Paz.

Palabras clave: transformaciones urbano-rurales, comunidades, análisis territorial

Abstract: hampaturi and Retamani are territories which display a constantly increasing articulation with the city of La Paz. Thus, these territories express a process of organization with does not include the administrative municipal limits of which they are a part. This article, based on tours and interviews, suggests an analysis of the spatial situation via a description of the transformations evidenced in the use of the land in both territories. It is linked to the use of land for agricultural production and how this is linked to different markets in the city of La Paz.

Keywords: urban-rural transformations, communities, territorial analysis

\footnotetext{
1 Este artículo es la suma de experiencias de trabajo en los lugares de estudio. Por un lado, se rescata la experiencia de trabajo en el distrito 22, Hampaturi, del municipio de La Paz con el "Proyecto Aprender Jugando 3: Ecoalfabetización desde la escuela hacia la comunidad para vivir bien en armonía con la Madre Tierra", de la Fundación Teko Kavi ejecutado por KINVULTUR S.R.L. en 2016 y, por otro lado, se compara el espacio de Hampaturi con los recorridos del mes de febrero de 2020 y 2021 realizados por la autora. Para el caso de Retamani, los ingresos corresponden al mes de febrero de 2020 solamente.

2 Carrera de Sociología, UMSA, La Paz. E-mail: bececarlandrea@gmail.com: ORCID: 00000002-2883-9911
} 


\section{INTRODUGGIÓN}

Hampaturi, a pesar de pertenecer administrativamente al municipio de La Paz, aún mantiene una estrecha relación con el municipio de Palca. Esta situación me ha permitido vincular a Hampaturi y a la comunidad de Retamani para la construcción del presente artículo. En mis recorridos por estos sectores, he encontrado que estos territorios comparten transformaciones desde lo urbano hacia lo rural, que son visibles desde el uso que se hace en el espacio (tanto para la producción de alimentos o por el cambio en el uso del suelo). Ambos territorios muestran ser influidos por su cercanía con diferentes zonas de la urbe paceña y por, sobre todo, por ser territorios proveedores de materias primas naturales. Así este artículo tiene como objetivo describir las transformaciones que se observan en ambos territorios y, a partir de ello, explicar las situaciones, conexiones y procesos que se expresan en la dinámica de la transformación territorial de Hampaturi y Retamani. Estos factores permiten el análisis de la interacción entre lo urbano y lo rural.

\section{LO URBANO Y LO RURAL EN EL ESTUDIO DEL TERRITORIO}

La literatura sobre los conceptos de lo urbano y lo rural ha sido trabajada por diversos autores y por sobre todo desde el enfoque del desarrollo. Lo urbano y lo rural se han categorizado de la siguiente manera:

Las sociedades "metropolitanas" de la Europa occidental y de Norteamérica [que] son los estados "avanzados", "desarrollados", industrializados; los centros del poder económico, político y cultural. En agudo contraste con ellas (...) están las demás sociedades, consideradas "subdesarrolladas": sociedades que continúan siendo principalmente agrícolas y "subindustrializadas". Los estados metropolitanos, mediante un comercio, pero también mediante un conjunto de controles económicos y políticos, extraen alimento y, lo que es aún más importante, materias primas, de esas regiones de suministro, esta eficaz "tierra interior" que ocupa además la mayor parte de la superficie terrestre y que contiene la gran mayoría de la población. (Williams 1973/2011: 345) (...) El texto de Raymond Williams citado arriba analiza las relaciones entre "el campo y la ciudad" en Inglaterra desde el siglo XVI hasta mediados del siglo XX, a través de la literatura, pero destacando las transformaciones económicas y políticas (Spedding, et al., 2013: 9).

La descripción que hace Williams sobre la relación del campo y la ciudad no ha cambiado en líneas generales. Los conceptos de lo urbano y lo rural señalan 
características que difícilmente se pueden vincular a las diversas realidades presentes en Bolivia. Esto ha sido representado para autores como Heredia (2016) que exista una "dicotomía urbano-rural". El autor señala que es dificil delimitar conceptualmente las fronteras que existen entre lo rural y lo urbano, ya que, éstas adquieren vida propia y producen escenarios característicos propios impregnados de cualidades dotadas por los sujetos sociales, "quienes construyen y redefinen los territorios y sus relaciones a partir de sus imaginarios colectivos, proyectos políticos, sus patrones socioculturales, sus intereses y estrategias económicas y el carácter de su territorialidad; influidos también por los factores del contexto globalizado, el mercado y la tecnología, entre otros fenómenos más externos" (2016: 14). Antequera Durán (2010) considera que las categorías de urbano, rural y periurbano deberían ser entendidas como relaciones sociales, ya que solo presentan dicotomías y contra posiciones en el imaginario. Este autor sostiene que las ciudades de Bolivia se han "andinizado", a través del "control máximo de pisos socioeconómicos y ya no solo ecológicos" (2010: 24). Para ello toma dos conceptos del manejo territorial del mundo andino, como es la doble residencia y el control vertical de los pisos ecológicos y desde los cuales se puede pensar en un modelo de urbanización generalizado a nivel país.

A través de estos conceptos es que Antequera Durán ha podido identificar que la relación social se da por medio de un ciclo agrícola-laboral-comercial, donde el trabajo temporal en ciudades permite incorporar la lógica del control de un máximo de pisos ecológicos sin perder los vínculos y las relaciones con la comunidad. Por ello, sostiene que "la periurbe en Bolivia es la continuación de la ruralidad a la vez que es su negación. Es, al mismo tiempo, la negación de lo urbano; es la no ciudad, pero junto a la ciudad" (ibid.: p. 36). Considero que hablar de periurbe como propone Antequera Durán sería limitado; más bien pienso que la noción de interfase urbano rural de López Pérez et al., (2005) permite reflexionar que estas "zonas de interfase son espacios geográficos en los que se dan distintas expresiones territoriales” (2005: 31), sistémicas a partir de la construcción de relaciones internas que reflejan una dimensión histórica en la que se pueden encontrar factores como "las interrelaciones, interdependencias e interconexiones que se producen entre los componentes internos (dinámica de población, calidad de vida y características geográficas, físico espaciales, entre otras)" (ibid.: 34), siendo aquí el lugar donde se produce la interfase, ya que las características del área rural y sus componentes naturales 
se reducen a las necesidades de la interfase urbano-rural. De esta manera la interfase urbano-rural se visibiliza a través del territorio en el que se asume implícitamente la existencia de un espacio geográfico y de un sujeto que ejerce sobre éste cierto dominio, una relación de poder, una calidad de poseedor o una facultad de apropiación, en la que los diferentes actores ejercen su territorialidad, construyendo con ello sus propios territorios diferenciados, donde pueden existir tantos territorios y territorialidades como actores que ejerzan dominio, que se haya apropiado, que se identifiquen con ella y defiendan su uso como construcción otorgada por los actores.

Dentro de los casos de estudio, la interfase urbano-rural ha sido trabajada por Le Gouill y Poupeau quienes, a través del análisis de las desigualdades en áreas periféricas de la zona Sur de La Paz, han hallado disparidades sociales y económicas entre los actores estudiados. Por un lado, se encuentran los comunarios, quienes son "agricultores" y por otro lado están los vecinos, quienes son los "llegados" 3 . Por ello han considerado que el mejor abordaje para la investigación es la categoría "territorial", debido a que el espacio es fuertemente segmentado y condiciona un conjunto de formas de vida. Otro punto de desigualdad está referido a la pertenencia organizacional entre comunarios y vecinos, lo que produce identidades múltiples con lógicas diferentes (cooperativistas, territoriales, profesionales, organizacionales, políticas, entre otras). (Le Gouill y Poupeau, 2020: 342). Estas desigualdades están estructuradas a partir de la "fortaleza de las formas de auto-organización del agua y de la integración a la ciudad" (ibid:: 343), siendo ante todo una definición por el territorio y de su articulación con el mundo exterior. Este aspecto muestra una relación de complementariedad, técnica o por contactos desde unos y del conocimiento territorial por los otros; pero esto forma parte de los juegos de poder a diferentes niveles de acción, pues a "nivel local, los comunarios movilizan sus derechos costumbristas y territoriales para compensar una situación histórica de exclusión" (ibid:: 347). Sin embargo, la urbanización de la zona Sur ha mostrado una constante mutación "y cada vez más fragmentando, social, política y organizacionalmente" (ibid.: 348). Este proceso desigual es afianzado por el gobierno municipal y nacional, particularmente por lo político.

3 Hace referencia a las personas que llegan por primera vez a esta zona para instalarse en sus viviendas y no forman parte de la comunidad. 


\section{LOS CASOS ESTUDIADOS}

\section{El territorio de Hampaturi}

Si bien el Illimani es un símbolo que representa a la ciudad de La Paz, los diferentes nevados de la Cordillera Real de los Andes que bordean a la ciudad vierten sus aguas hacia la zona de Hampaturi, donde forman varias lagunas y ríos que dan vida a "las cabeceras de las cuencas Choqueyapu, Irpavi, Achumani y Orkojahuira que conforman la macrocuenca del Río La Paz" (GAMLP, 2009: 4). Hampaturi es un territorio que ha sido proyectado para la provisión de agua, sus "represas, (...) y sus aducciones, [fueron] instaladas en 1930-1940" (Torrico, cit. en Le Gouill y Poupeau, los corchetes son míos), y desde el 2016 se ha intensificado su rol de proveedor con la construcción de una represa de agua que sirva de abastecimiento principalmente a la zona Sur ${ }^{4}$ de la ciudad de La Paz. Hampaturi fue un sector de hacienda y los comunarios "han adquirido sus títulos de propiedad luego de la Reforma Agraria de 1953, a través del Instituto de Reforma Agraria, y están oficialmente vinculados al municipio de Palca" (Le Gouill y Poupeau, 2020: 343). Sin embargo, desde 1995 a través de la Ley 1669 (Producción Agrícola Sostenible, PROAGRIS, 2012: 4) Hampaturi administrativamente forma parte de los dos macrodistritos rurales que pertenecen al municipio de La Paz.

El Gobierno Autónomo Municipal de La Paz (GAMLP) señala que el macrodistrito de Hampaturi comprende las áreas limítrofes del este y norte de la ciudad; limita con los municipios de Palca, Coroico y Pucarani. Además, tiene como límite al Parque Nacional y Área Natural de Manejo Integrado (PN-AMNI) Cotapata. El territorio de Hampaturi tiene una extensión de 445 km y "constituye el 22\% de la superficie del municipio de La Paz" (GAMLP, 2007: 34). El municipio ha considerado a Hampaturi como una "zona de expansión de la mancha urbana, debido a la vinculación con la dinámica urbana, en especial la zona de Cheka-Chinchaya" (GAMLP, 2009: 9). Las comunidades de Cheka-Chinchaya, Muramaya, Lorocota y Alpacoma son las que presentan una fuerte continuidad del asentamiento poblacional nuclear (figura 1) como extensión de los barrios de Villa Salomé y Pampahasi (figura 2). El acceso carretero fue construido paralelamente a la represa, que es conocida por los comunarios

4 La investigación de Perales (2018) sobre la “crisis del agua” de 2016 señala que afectó a 94 barrios de la ciudad de La Paz, que fueron desabastecidos o racionados por un periodo de tres meses. En su mayor parte, esto barrios se encontraban en la zona Sur de la ciudad de La Paz. 
Figura 1. Comunidades de Cheka Chinchaya y Chicani, dentro del macrodistrito de Hampaturi.

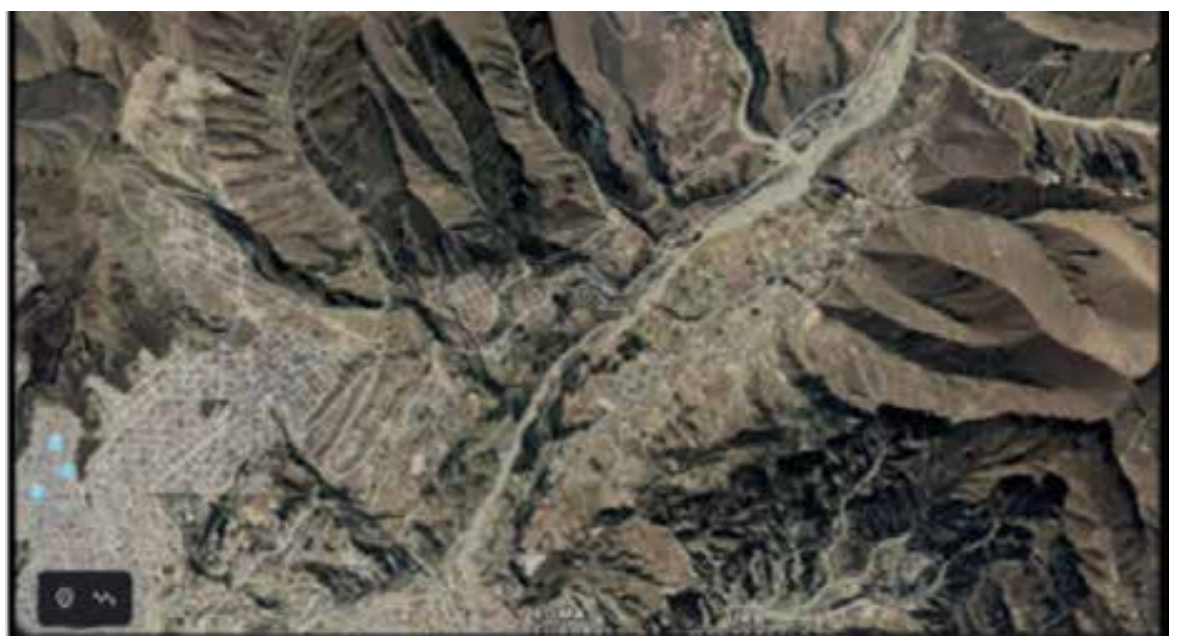

Fuente: Gooogle Earth, 2020.

Figura 2. Imagen satelital de las comunidades de Cheka Chinchaya y Chicani como continuidad de los barrios urbanos de la ciudad de La Paz.

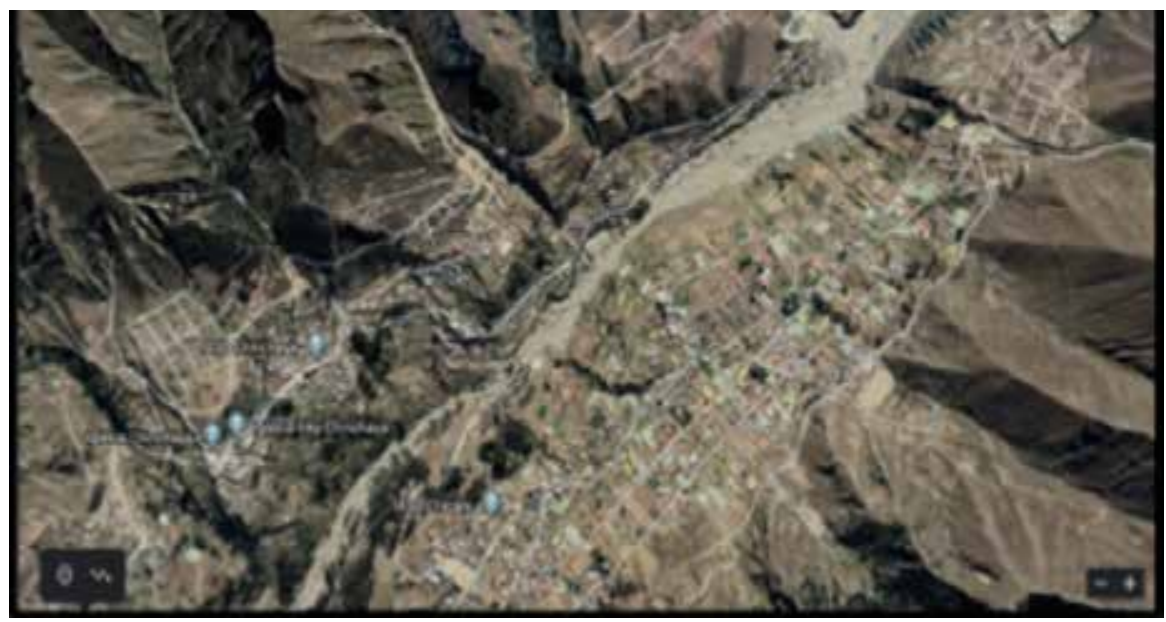

Fuente: Gooogle Earth, 2020. 
como "La Pequeña" (Torrico, 2020: 363). En 2017, fue extendido hasta la zona alta, donde se construyó la segunda represa denominada "La Grande" (ibid.). A la comunidad de Chicani se puede acceder por esta carretera. También esta comunidad puede conectarse hacia la zona sur desde Irpavi. Tanto vecinos como comunarios de Cheka-Chinchaya utilizan esta vía tanto para salir hacia Villa Salomé como hacia Irpavi. El tiempo estimado de viaje en un coche particular es de 45 minutos. El transporte hacia las comunidades es realizado por los comunarios; sin embargo, no se cuenta con una línea de transporte público.

Desde el ingreso por Villa Salomé se encuentran las comunidades de Cheka-Chinchaya, Muramaya, Chicani, Lorocota y Alpacoma. Cada comunidad tiene una escuela, una sede y una cancha. En la escuela, en la sede como en la avenida principal, se observa una fuerte presencia del GAMLP. Mientras tanto, en las canchas, así como en algunas de las viviendas ${ }^{5}$ y en los proyectos de agua se exhiben los colores y el logo del gobierno central como una muestra de su presencia institucional. Su nacimiento como un territorio proveedor de agua ha hecho que Hampaturi sea reconocido -tanto por el municipio como por el gobierno central- por esta característica, que ha cobrado una mayor significación durante la segunda década del siglo $\mathrm{XXI}^{6}$. Según una informante, este relacionamiento con las instituciones gubernamentales -tanto municipales como centrales- ha posibilitado que la organización social de las comunidades haya replanteado su ejercicio del poder, al momento de proporcionar un acceso para proveer de agua hacia la ciudad. Es decir, el desabastecimiento que sufrió la ciudad en 2016, representó una oportunidad para obtener mayor cantidad de proyectos - principalmente de infraestructura- para que fueran desarrollados dentro de las comunidades. Las construcciones de viviendas nuevas hechas con ladrillo y cemento se mezclan con las pocas casas de adobe que muestra el paisaje (figura 3). En las comunidades, desde septiembre de 2016 hasta febrero de 2021, se ha intensificado la venta de casas o de lotes -particularmente, aquellos cercanos

\footnotetext{
5 Me refiero a aquellas que han sido construidas dentro de las viviendas sociales y que son rápidamente reconocidas porque sus paredes exteriores están pintadas de blanco y sus techos son de calamina azul.

6 La literatura sobre Hampaturi y su rol de proveedor de agua potable para la ciudad de La Paz, ha sido ampliamente trabajada por Hardy (2013), Le Gouill y Poupeau (2020), Perales Miranda (2018), Urquieta (2019), Torrico Foronda (2020).
} 
a las zonas de Villa Salomé o de Irpavi- que se encuentran en la avenida principal o en accesos secundarios que están a una distancia estimada de 10 a 15 minutos a pie hacia la avenida. En la avenida principal de Chinchaya y Chicani es interesante observar que las viviendas en su mayoría tienen pequeñas a medianas carpas solares y un espacio de corral para diferentes tipos de animales domésticos (figura 4).

La formación geológica de Hampaturi se compone de "una serie de rocas aluviales, de diferentes dimensiones" (Becerra Cardona et al., 2016: 29); esta característica ha permitido que los comunarios hayan implementado una canalización para la extracción de piedra, grava y arena. La extracción de áridos es realizada a través de canales donde se han desbordado ríos, riberas y arroyos para que sus aguas se dirijan a una especie de pozo para soltar el material del suelo. El lavado para la extracción de áridos se realiza generalmente durante la época de lluvias, porque su proceso requiere de grandes cantidades de agua, que son necesarias para obtener una mejor calidad de arena. Después de haber lavado y separado la piedra, la grava y la arena, las aguas son reencausadas "a su lecho original, hasta que llegue a otra cuadrícula de aprovechamiento de arena o grava" (ibid.). La figura 5 muestra las modificaciones que sufren los caudales ecológicos de los afluentes que recorren las comunidades. La extracción continua de áridos a pequeña escala puede provocar "impactos ambientales locales que pueden llegar a convertirse en impactos mayores, puesto que de la tierra lavada suele quedar sedimento fino que no tiene uso y se arrastra junto con el agua, lo que provoca las des-estructuraciones del suelo y mazamorras" (ibid.). Esta des-estructuración de las capas de suelo es visible en las aguas que se encuentran estancadas de "color café lechoso, por la cantidad de sedimentos que se arrastran producto de la actividad de extracción de áridos" (ibid.). Los áridos son comercializados en obras de construcción en la ciudad de La Paz, la figura 6 es un ejemplo de la maquinaria pesada que poseen los comunarios para su traslado. Así, la extracción de áridos es una de las principales actividades económicas del sector. 
Figura 3. Viviendas de la comunidad de Cheka Chinchaya, que muestran la cercanía de unas con otras y los materiales con los cuales fueron edificados

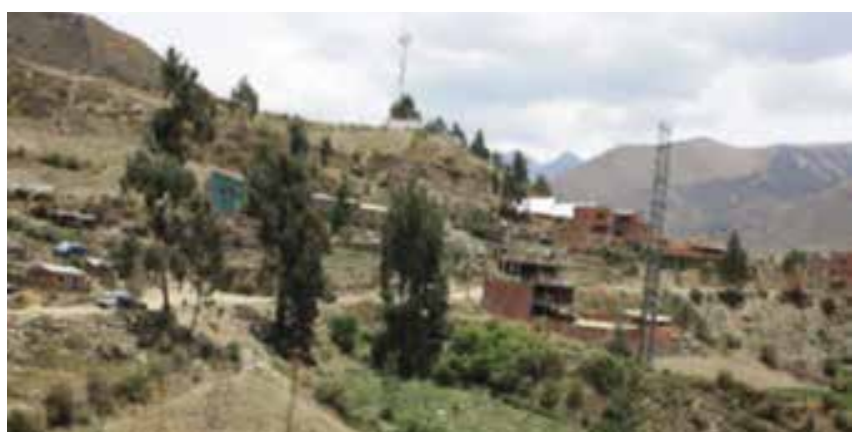

Fotografia: Carla Becerra.

Figura 4. Vivienda de Cheka Chinchaya donde puede observarse el corral, la carpa solar, el corral y la casa familiar, lado a lado

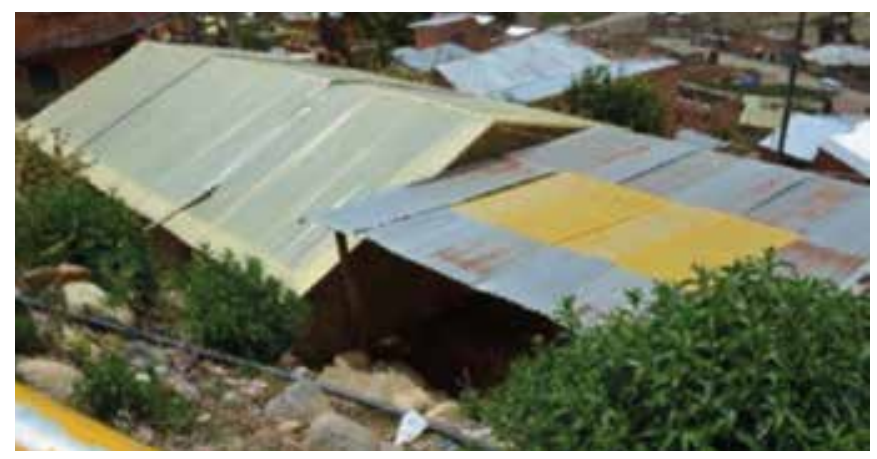

Fotografia: Mirtha Velásquez. 
Figura 5. Modificaciones al caudal ecológico, para extracciones de arena

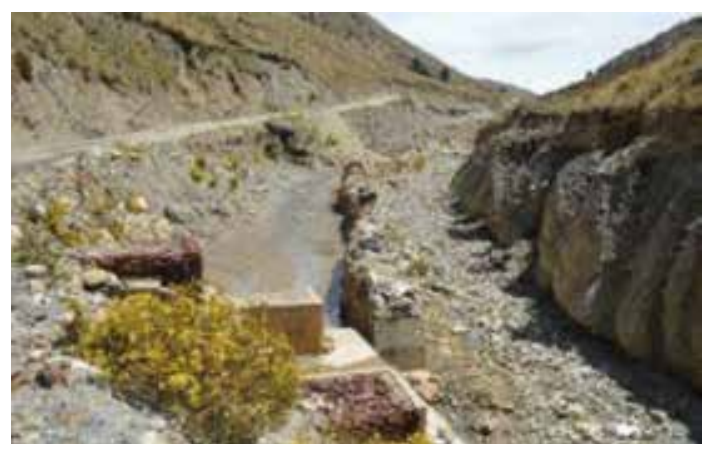

Fotografia: Heiddy Mallea.

Figura 6. Volqueta ubicada en la casa de un comunario, cerca al río

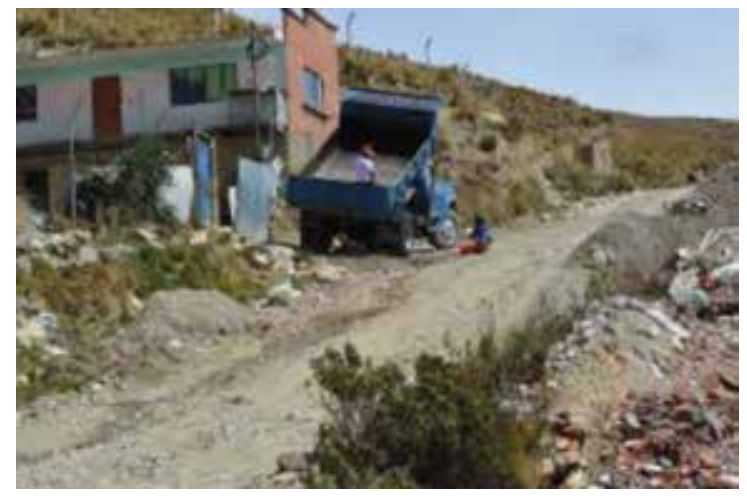

Fotografia: Heiddy Mallea.

Debajo de la nueva represa, hacia el lado izquierdo, puede observarse aguas con contenido de copajira, que presentan una coloración rojo cobrizo a causa de la mina de donde se extraía wolfrang, que se encuentra al lado derecho y que pertenecía a la empresa llamada "La Solución". En 2021, las cascadas que se encuentran debajo de la represa y esta mina se han convertido en un atractivo turístico. El gran agujero que se encuentra debajo de la mina y visible a un costado de la montaña muestra paredes 
teñidas de copajira; las gotas de agua con este mineral que caen hacia el suelo forman un pequeño riachuelo que luego es captado en tubos blancos que atraviesan y marcan las veredas de los cerros que siguen hasta perderse de vista hacia la comunidad de Lorocota. A su costado, paralelamente se encuentran ubicados los tubos que sirven de afluentes para la transformación de agua potable a la ciudad. Sin embargo, el paisaje de Hampaturi permite mitigar "hasta cierto grado, por la función de filtración que cumplen los restos o vestigios de bofedales que se encuentran en ciertos lugares" (ibid.), las aguas con sedimento y con contenido de copajira, aunque la expansión de la ciudad de La Paz, "la construcción de caminos, (...) la extracción de turba" (ibid.) y el creciente turismo colocan en riesgo a estos ecosistemas.

\section{El municipio de Palca}

Ahora pasaré a describir al municipio de Palca (figura 7), situado a 50 kilómetros de la sede de gobierno. Limita al este con la provincia Sud Yungas, al sur con la provincia Loayza y el municipio de Mecapaca, al oeste con la ciudad de La Paz (zonas Villa Salomé, Irpavi, Achumani y Chasquipampa) y Mecapaca al norte con la ciudad de La Paz y el municipio de Yanacachi. El principal acceso carretero hacia el centro de Palca es a través de la salida de las zonas de Ovejuyo y Chasquipampa de la parte sur de la ciudad de La Paz, pasando por Uni y el Valle de las Ánimas. De allí hacia el poblado de Palca se tarda unos 30 a 40 minutos en llegar y poco a poco el camino deja de mostrar viviendas aglomeradas, hasta encontrarse con un paisaje que muestra los primeros cultivos.

El ingreso a la comunidad de Retamani (figura 8), se realiza por la parte sur; esta comunidad muestra a varias familias que se encuentran cercanas hacia el poblado de Palca, a pocos minutos de distancia. Esta comunidad tiene un sindicato agrario y se caracteriza por la producción agrícola, donde cinco unidades domésticas han apostado por la práctica de la agricultura favorable y amigable con el medio ambiente para poder mantener el suelo. En la última década hay una fuerte incursión de los varones en el transporte público - desde las comunidades hacia la calle Benancio Burgoa, circundante al mercado Rodríguez de la ciudad de $\mathrm{La} \mathrm{Paz}^{-}$y ello ha hecho que se convierta en una de las principales fuentes económicas de esta localidad. 
Durante la gestión 2018, la crecida del río Palca ${ }^{7}$ arrasó con los puentes de ingreso al hospital y a la represa construida en la zona. Se destruyeron 60 viviendas construidas a la vera del río, además se perdieron sembradíos de papa, choclos, hortalizas, entre otros, afectando a 150 familias en todo el municipio de Palca (Correo del Sur, 15-2-2018).

Figura 7. Municipio de Palca

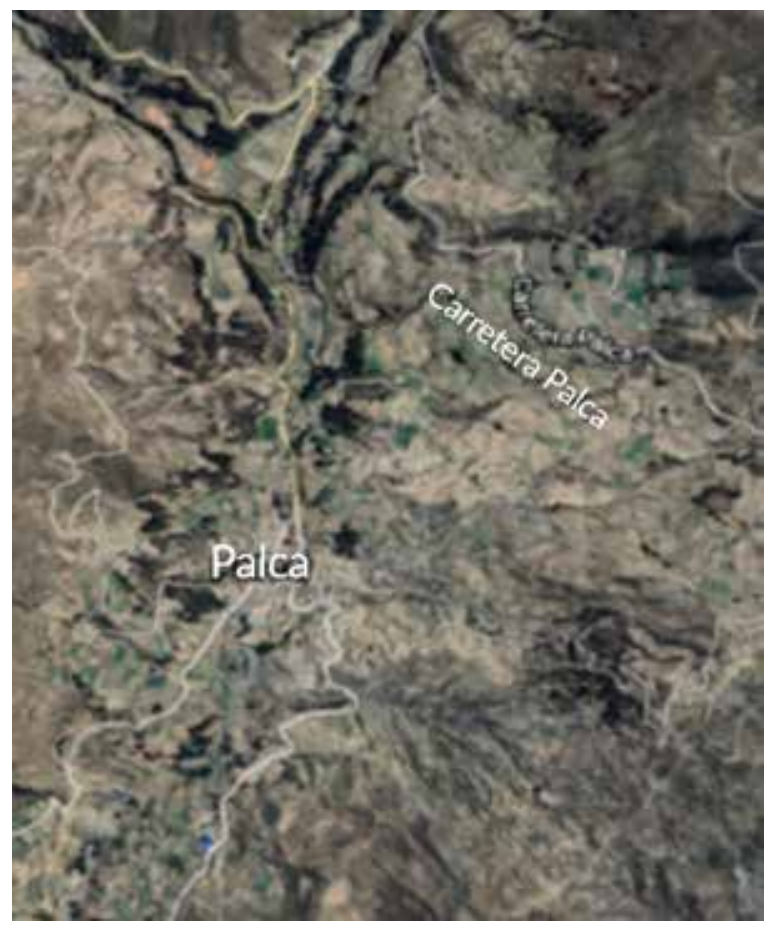

Fuente: Gooogle Earth, 2020.

7 Para más información, véase las notas de prensa de 2018 sobre el desborde del río Palca (Los Tiempos, 15-2-2018; Agencia de Noticias Fides, ANF, 15-2-2018; Flores, 16-2-2018). 
Figura 8. Comunidad Retamani del municipio de Palca

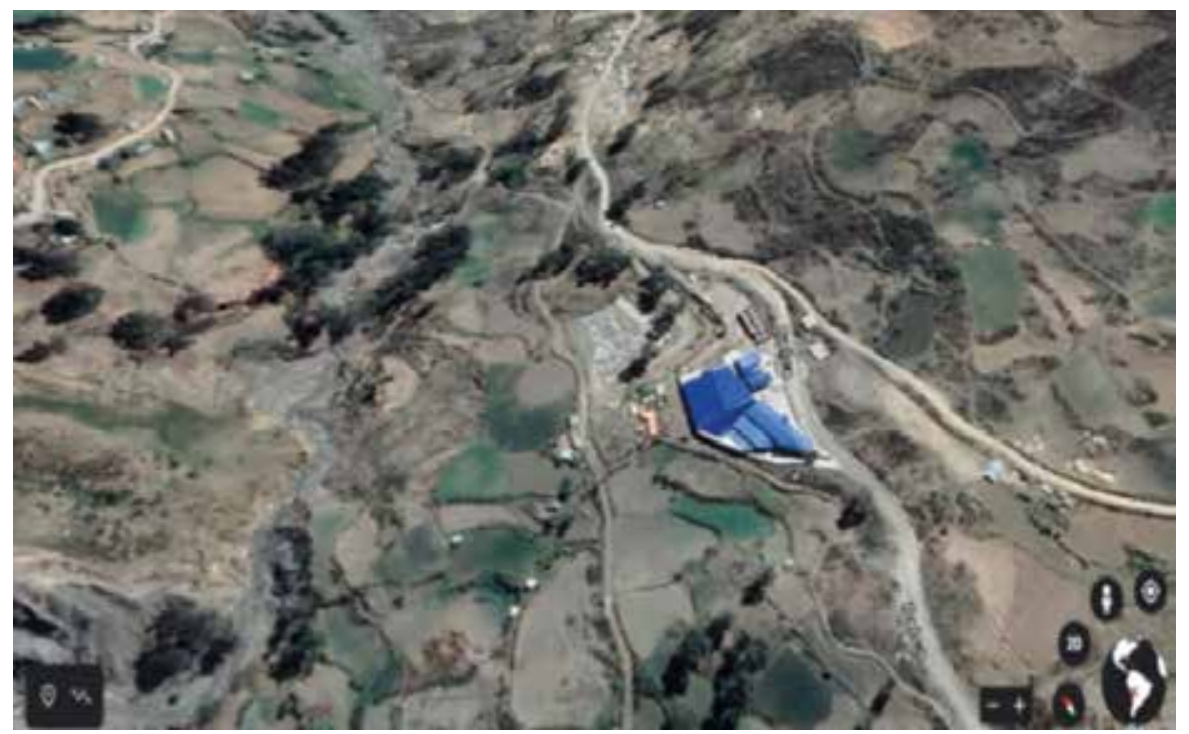

Fuente: Gooogle Earth, 2020.

Este catastrófico desastre natural marcó la vida de los pobladores, ya que, a partir de ello, cada familia tuvo que proveerse de agua mediante politubos hacia sus domicilios (entrevista a Andrés Chambi Quispe, 15 de febrero de 2020). El recurso hídrico se utiliza principalmente para la producción agrícola, por medio de acequias que proveen de agua para riego a los cultivos. Los comunarios han informado que las tierras se encuentran saneadas por el INRA y que durante el saneamiento de tierras se ha dotado por igual tanto a varones como a mujeres. También hay la venta de lotes de terreno que pueden ser comprados por miembros de la misma comunidad, comunidades vecinas o foráneos. Sobre este último se pudo conocer sobre la venta de un terreno donde se construyó un centro de retiro de una iglesia evangélica.

\section{EL USO DE SUELO PARA PRODUCGIÓN ECONÓMICA}

Los propietarios de las carpas solares de Hampaturi comercializan sus productos en mercados o ferias. En la ciudad de El Alto se puede acceder 
a estos productos en la zona de Villa Dolores y en la ciudad de La Paz en las zonas de Villa Copacabana y Pampahasi. De acuerdo a los testimonios de los pobladores, las carpas solares se mantienen a través del fomento a la vocación rural que promueve el municipio de La Paz en la zona para motivar a los productores a una buena producción agrícola, a pesar de que gran parte del suelo agrícola cada vez muestra encontrarse en una interfase rural-urbana. En estas carpas solares, se producen una serie de hortalizas, hierbas aromáticas y medicinales, entre otras. Cultivos de papa, haba, oca, entre otras es producido sobre los cerros circundantes a los accesos carreteros, debido a la extensión de tierra que se requiere; pero también es posible observar esta producción en lotes de terreno amurallados. En las comunidades es posible divisar especies forestales introducidas como el eucaliptus y pino; en la parte superior y cercana a las cascadas se observa kiswaras (cuadro 1).

Cuadro 1. Especies de flora observada en Hampaturi

\begin{tabular}{|l|l|l|l|l|l|}
\hline \multicolumn{5}{|l|}{ Especies cultivadas } & Especies forestales \\
\hline $\begin{array}{l}\text { Nombre } \\
\text { común }\end{array}$ & $\begin{array}{l}\text { Nombre } \\
\text { científico }\end{array}$ & $\begin{array}{l}\text { Nombre } \\
\text { común }\end{array}$ & $\begin{array}{l}\text { Nombre } \\
\text { científico }\end{array}$ & $\begin{array}{l}\text { Nombre } \\
\text { común }\end{array}$ & $\begin{array}{l}\text { Nombre } \\
\text { científico }\end{array}$ \\
\hline Papa & $\begin{array}{l}\text { Solanum } \\
\text { Tuberosum spp. }\end{array}$ & Ilusión & $\begin{array}{l}\text { Gypsophila } \\
\text { spp. }\end{array}$ & Eucalipto & $\begin{array}{l}\text { Eucaliptus } \\
\text { globulus }\end{array}$ \\
\hline Papaliza & Ullucus tuberosus & Rosa & Rosa spp. & Pino & Pinus spp. \\
\hline Haba & Vicia faba & Margarita & Bellis perennis & kiswara & Buddleja \\
\hline Oca & Oxalis tuberosa & Dalia & Dahlia spp. & & \\
\hline Arveja & Pisum sativum & Gladiolo & Gladiolus spp. & & \\
\hline Nabo & Brassica rapa & Tulipanes & Tulipa spp. & & \\
\hline Zanahoria & Daucus carota & Clavel & $\begin{array}{l}\text { Dianthus } \\
\text { caryophyllus }\end{array}$ & & \\
\hline Cebada & Hordeum vulgare & Cartucho & $\begin{array}{l}\text { Zantedeschia } \\
\text { aethiopica }\end{array}$ & & \\
\hline Cebolla & Allium cepa & & & & \\
\hline Repollo & $\begin{array}{l}\text { Brassica } \\
\text { oleracea }\end{array}$ & & & & \\
\hline Lechuga & Lactuca sativa & & & & \\
\hline
\end{tabular}




\begin{tabular}{|l|l|l|l|l|l|}
\hline Perejil & $\begin{array}{l}\text { Petroselinum } \\
\text { crispum }\end{array}$ & & & & \\
\hline Apio & Apium graveolens & & & & \\
\hline Acelga & Beta vulgaris & & & & \\
\hline Choclo & Zea mays & & & & \\
\hline Zapallo & Cucurbita maxima & & & & \\
\hline Cedrón & Aloysia citrodora & & & & \\
\hline Achojcha & Cyclantherapedata & & & & \\
\hline Huacataya & Tagetes minuta & & & & \\
\hline Tomillo & Thymus & & & & \\
\hline Ciruelo & Prunus domestica & & & & \\
\hline Uva & Vitis & & & & \\
\hline
\end{tabular}

Fuente: Elaboración propia, con base a Becerra Cardona, et al. (2016: 31 ) y las observaciones durante el recorrido de 2020 y 2021.

Sobre la cría de ganado vacuno, ovino y camélido, los informantes señalan la cría de vacas lecheras cuyos productos son comercializados tanto en ferias, como a visitantes y a las principales fábricas como PIL y Delizia. El ganado camélido ha sufrido debido a la construcción de la represa, ya que en este lugar se encontraban bofedales aptos para su pastoreo, lo que, según nuestro informante, habría conllevado a que los adultos mayores que aún se dedican a esta actividad deban ir más lejos con estos animales. También se ha observado la actividad de producción avícola.

Por su parte, la característica de Retamani es la producción agrícola a campo abierto, esto se debe a que en la zona la tenencia de tierra abarca unas 5 hectáreas por comunario. Como se ha mencionado, cinco familias de la comunidad de Retamani practican la agricultura amigable con el medio ambiente. Es de interés mencionar que los comunarios han señalado repetidamente que de la producción que realizan solo comercializan los productos que tienen un mercado seguro; esto se debe a que los mercados de Chasquipampa y Rodríguez de la ciudad de La Paz tiene intermediarios, quienes regulan la oferta y demanda del mercado, así como los puestos de venta en el mismo (cuadro 2). Sin embargo, otro factor que los entrevistados señalan es el aspecto de los alimentos al momento de la compra y el contra- 
bando. Ellos señalaron que, debido al regateo que hacen los compradores al momento de comprar los productos, la principal característica que observan son las abolladuras y marcas. Estas características definen el precio del producto en el mercado.

Cuadro 2. Especies de flora observada en Retamani

\begin{tabular}{|c|c|c|c|c|c|}
\hline \multicolumn{4}{|c|}{ Especies cultivadas } & \multicolumn{2}{|c|}{ Especies introducidas } \\
\hline $\begin{array}{l}\text { Nombre } \\
\text { común }\end{array}$ & $\begin{array}{l}\text { Nombre } \\
\text { científico }\end{array}$ & $\begin{array}{l}\text { Nombre } \\
\text { común }\end{array}$ & $\begin{array}{l}\text { Nombre } \\
\text { científico }\end{array}$ & $\begin{array}{l}\text { Nombre } \\
\text { común }\end{array}$ & $\begin{array}{l}\text { Nombre } \\
\text { científico }\end{array}$ \\
\hline Papa & $\begin{array}{l}\text { Solanum } \\
\text { Tuberosum } \\
\text { spp. }\end{array}$ & $\begin{array}{l}\text { Pompones } \\
\text { amarillos }\end{array}$ & Dahlia spp. & Eucalipto & $\begin{array}{l}\text { Eucaliptus } \\
\text { globulus }\end{array}$ \\
\hline Haba & Vicia faba & $\begin{array}{l}\text { Pompones } \\
\text { blancos }\end{array}$ & Dahlia spp. & Pino & Pinus spp. \\
\hline Arveja & $\begin{array}{l}\text { Pisum } \\
\text { sativum }\end{array}$ & & & & \\
\hline Nabo & $\begin{array}{l}\text { Brassica } \\
\text { rapa }\end{array}$ & & & & \\
\hline Cebolla & Allium cepa & & & & \\
\hline Repollo & $\begin{array}{l}\text { Brassica } \\
\text { oleracea }\end{array}$ & & & & \\
\hline Lechuga & $\begin{array}{l}\text { Lactuca } \\
\text { sativa }\end{array}$ & & & & \\
\hline Choclo & Zea mays & & & & \\
\hline Tumbo & $\begin{array}{l}\text { Passiflora } \\
\text { tripartita }\end{array}$ & & & & \\
\hline $\begin{array}{l}\text { Carote/ } \\
\text { zucchini }\end{array}$ & $\begin{array}{l}\text { Cucurbita } \\
\text { pepo subsp. } \\
\text { pepo }\end{array}$ & & & & \\
\hline $\begin{array}{l}\text { Beterraga/ } \\
\text { Remolacha }\end{array}$ & Beta vulgaris & & & & \\
\hline
\end{tabular}

Fuente: Elaboración propia con base en las observaciones durante el recorrido del 15 de febrero de 2020. 
Los comunarios de Retamani indican que, en tiempo de baja demanda de sus productos, ellos han optado como medida y estrategia de vida el consumo de su producción; también mantienen la práctica de siembra en familia. Esto último es visto por nuestros informantes como una medida de prevención, pues señalan "que sus hijos sabrán sembrar y ser profesionales”. En el municipio de Mecapaca aún se utiliza la yunta para el arado de la tierra. Debido a la incursión de la ONG Caritas en la comunidad de Retamani se ha promovido el uso de takanas para la siembra de productos como cebolla y carotes, etc. Estas familias utilizan fungicidas naturales a partir de mezclas entre orín, locoto, tabaco, ceniza entre otros, para combatir las enfermedades que puedan aparecer en los cultivos. Los esfuerzos por realizar producciones amigables con la naturaleza no son apreciados al momento de competir en el mercado, como se ha descrito anteriormente.

Sin embargo, queremos hacer notar el rol de los intermediarios en relación con los productores. Nuestros informantes señalan que, una vez listos los cultivos para su cosecha, las familias acopian el producto al costado del camino para el recojo de los productos por un minibús que es conducido por otro comunario o por uno propio, los días martes y viernes, con dirección al mercado de Villa Dolores en la ciudad de El Alto para llegar a éste a las cinco de la mañana, hora en la que comienzan los productores a ofertar sus diversos productos a los intermediarios. Carla, una productora-comerciante, nos explicaba que la oferta de la venta de cebolla se encuentra sujeta al contrabando del producto peruano, puesto que nuestros productores clasifican el producto de la cebolla por tamaño, categorizándolo así de primera, segunda y tercera, ganando así por chipa (cesto) mientras que la cebolla comercializada del Perú es vendida por saka$\tilde{n} a$ (saquillo). Esta situación ha promovido que ellos consideren que el cultivo de cebolla "baratee". Esta intervención de los mayoristas y detallistas ha provocado que el productor se someta al contrabando, situación que no solo tiene un efecto a nivel microsocial, sino también a nivel macrosocial, 
reflejado en los precios de la canasta familiar ${ }^{8}$. Otro aspecto importante a señalar es la postura que muestran sobre la denominación de "producción ecológica". Modesto fue contundente al indicar que "nadie somos ecológicos, natural sí" (entrevista, 15-2-2020), pues considera que no hay una producción ecológica, ya que el municipio muestra diversos patrones de uso antrópico como el transporte o los usos de plaguicidas, fertilizantes y funguicidas por otros productores, lo que afecta a las producciones. Por ello es que Modesto lo considera natural y no "ecológico".

\section{REFLEXIONES FINALES}

Señalaré que ambas zonas descritas tienen una fuerte relación con lo urbano, pues a partir de su nexo han construido y desarrollado "dinámicas (...) según sus objetivos, proyectos e imaginarios. Eso constituye una de las fuentes de identidad y funcionalidad de los espacios geográficos como unidades socioterritoriales, cohesionadas y complementadas por fronteras dinámicas, difusas, complejas y con vida propia" (Heredia, 2016: 71). En concordancia con Henry Lefebre y Silvia Rivera (citados en Dolph, 2010: 95) el campo y la ciudad entran en una relación dialéctica donde el campo en relación con la ciudad extrae riqueza del campo y la concentra en la ciudad para engendrar una relación desigual. Sin embargo, para fines de este artículo se tiene que diferenciar las relaciones que estas zonas mantienen con las urbes de La Paz y El Alto, pues la transformación sobre un territorio como el de Hampaturi es visible desde el espacio físico, mientras que en Retamani esto se muestra en la relación que tiene con el mercado. Pero, considero que en ambos casos existe una violencia simbólica ${ }^{9}$ de lo urbano hacia lo rural, a

8 El método que se utiliza para establecer los precios de la canasta familiar consiste en enviar a encuestadores "a los lugares de compra habituales de los consumidores previamente seleccionados, para capturar los precios de contado efectivamente transados en el mercado durante el período de referencia (el mes, en la mayoría de los casos)" (Sánchez, 2002: 45). La información que se recibe del mercado no refleja de dónde provienen los productos que se ofertan, simplemente se limita a recabar el costo para establecer así la canasta familiar. De esta manera, se deja a un lado el impacto del contrabando no solo para los productores nacionales.

9 Para Bourdieu y Wacquant, se puede entender como violencia simbólica a "esta forma de violencia que se ejerce sobre un agente social con su complicidad" (citado en Peña Collazos, 2009). 
través de "sutiles prácticas discriminatorias y las complejas relaciones que se tejen entre un conjunto de actores a partir de una superposición de intereses" (Carman, 2007: 11), no solo en el territorio como tal, por el crecimiento de los límites urbanos hacia los rurales como una forma de extensión, visualizada en Hampaturi y también desde el espacio urbano en el que se comercializan los productos, impactando directamente en el uso del suelo para cultivo, lo que tiene, además, un efecto económico.

En Hampaturi, se observa esta transformación territorial denominada "interfaz urbano-rural", pues este distrito alberga a más habitantes en una suerte de extensión de las zonas de Villa Salomé y Pampahasi en la parte norte-este y desde Irpavi en el sur de la ciudad de La Paz. En estos sectores, puede notarse ocupaciones del espacio y "diversas formas de manifestación cultural, organización, económica y productiva” (Antequera Durán, 2010: 23), que claramente responden a patrones propios, por un lado, desde el capital económico y, por el otro, en función del capital cultural sobre la posesión de la tierra como vivienda, lo que lleva a una gradual expansión urbana. Si bien en Retamani no se muestra una ampliación de lo urbano a lo rural, por medio de los entrevistados, se ha podido describir la recurrencia de su relación con los principales mercados de las ciudades de La Paz y El Alto. Además, las parejas jóvenes han salido de las comunidades de Palca para asentarse en zonas próximas a la ciudad o dentro de la misma, reflejando con ello este sentido de lo periurbano como ruptura y articulación que permite la multilocalidad para un mayor control de pisos ecológicos y económicos, como señala Antequera Durán, en su texto Itinerarios urbanos: continuidades y rupturas urbano rurales (2010).

Sin embargo, considero que lo descrito me permite reflexionar acerca de la "expulsión simbólica tanto de la naturaleza como de la cultura" de los asentamientos originarios, manifiestos desde la ciudad hacia lo rural, más allá de lo mencionado por Tassi y Canedo sobre las transformaciones del sistema campesino (2019: 47) desde la diversificación de actividades económicas. Creo que la ciudad juega sobre la ruralidad una violencia simbólica manifestada, por un lado, desde las diferencias morales que se promueven a través de la cultura alimenticia, donde se superponen los intereses de los ciudadanos urbanos sobre los productores y, por otro lado, en la tenencia de tierras, donde el acceso al espacio urbano "justifica toda arbitrariedad" desde lo público a través del Estado, tanto en sus niveles municipales como 
estatales. Bajo la noción de que el derecho al agua es un derecho humano, se ha producido lo que podríamos denominar una "gradación de humanidades" (Carman, 2007), bajo el entendido de que la condición de humanidad no implica el reconocimiento de lo humano sino que se produce un simétrico reconocimiento "unilateral de la sobrehumanidad de los que merecen un estilo de vida aparte" (ibid.: 22). Con esto quiero ejemplificar cómo he entendido lo observado en Hampaturi y Retamani, pues sostengo que lo expuesto se acerca no solo a la desigualdad descrita por Le Gouill Poupeau (2020), sino que también se materializa en el valor agregado del suelo para lo urbano y en la producción agrícola en los mercados.

\section{BIBLIOGRAFÍA}

Agencia de Noticias Fides (ANF) (15 de febrero de 2018). "Desborde de río causa destrozos en Palca y alcalde pide ayuda". Los Tiempos [sección Actualidad]. Recuperado de https://www.lostiempos.com/actualidad/ pais/20180215/desborde-rio-causa-destrozos-palca-alcalde-pide-ayuda Antequera Durán, Nelson (2010). "Itinerarios urbanos. Continuidades y rupturas urbano rurales". En Nelson Antequera Durán y Cristina Cielo, Ciudad sin fronteras: sobre la multilocalidad de lo urbano en Bolivia (pp. 23-40). La Paz: RITU Bolivia/Gobierno Autónomo Municipal de La Paz/CIDES-UMSA/ Fundación PIEB/Oxfam GB/Universidad de California Berkeley.

Becerra Cardona, Carla Andrea; Heiddy Asunción Cárdenas Mallea, Franklin Romeo Huajllari Fernandez y Mirtha Escarlen Velásquez Ramírez (2016). Estudio etnográfico de Hampaturi del municipio de La Paz. Proyecto Aprender Jugando 3: "Ecoalfabetización desde la escuela hacia la comunidad para vivir bien en armonía con la Madre Tierra". La Paz: Fundación Teko Kavi ejecutado por KINVULTUR S.R.L.

Carman, María (2007). "Las fronteras de lo natural y las fronteras de lo humano. En Instituto Nacional contra la Discriminación, la Xenofobia y el Racismo (INADI), Investigaciones por la diversidad, Investigaciones por la diversidad (pp. 11-29). Buenos Aires: INADI Ministerio de Justicia, Seguridad y Derechos Humanos. Presidencia de la Nación). Recuperado de https:// www.academia.edu/27875427/Las_fronteras_de_lo_natural_y_las_fronteras_de_lo_humano 
Chambi, Quispe Andrés (15 de febrero de 2020). Entrevista colectiva comunario de Retamani. Entrevistador: Carla Andrea Becerra Cardona, entrevistador. Correo del Sur (15 de febrero de 2018). "La Paz: crecida del río en Palca arrasa con 60 viviendas y afecta a unas 150 familias". Correo del Sur. Recuperado de https://correodelsur.com/sociedad/20180215_la-paz-crecida-delrio-en-palca-arrasa-con-60-viviendas-y-afecta-a-unas-150-familias.html Dolph, Charles (2010). "De la contradicción al continuum urbano rural. La urbanización, el legado colonial y la cultura de la democracia". En Nelson Antequera Durán y Cristina Cielo, Ciudad sin fronteras: sobre la multilocalidad de lo urbano en Bolivia (pp. 93-110). La Paz: Universidad de California/RITU Bolivia.

Flores, Rolando (16 de febrero de 2018). "Riada en Palca dejó 309 familias afectadas". El Diario [sección Noticias]. Recuperado de https://www.eldiario.net/noticias/2018/2018_02/nt180216/nacional.php?n=27\&-riada-en-palca-dejo-309-familias-afectadas

Gobierno Autónomo Municipal de La Paz, GAMLP (2010). Programa de Gobierno 2010-2015: las 10 Tareas para La Paz. La Paz: GAMLP. La Paz.

GAMLP (2009). "Jayma Rural”, Plan para el Desarrollo de las Áreas Rurales del Municipio de La Paz. La Paz: Dirección Especial para el Desarrollo, Unidad Espaecial de Planificación y Proyectos, Área de Desarrollo Rural, GAMLP.

GAMLP (2007). Resumen Ejecutivo Plan de Desarrollo Municipal. La Paz: Dirección de Planificación y Control, Unidad de Planificación Participativa, GAMLP.

Hardy, Sebastien (2013). Atlas de la vulnerabilidad de la aglomeración de La Paz. La Paz: Plural editores.

Heredia, Luis Fernando (coord.) (2016). Desdibujando fronteras: relaciones urbanas-rurales en Bolivia. La Paz: Centro de Investigación y Promoción del Campesinado.

Le Gouill, Claude y Franck Poupeau (2020). "Auto-organización de la gestión del agua y desigualdades: la zona Sur de La Paz frente a las transformaciones urbanas". En Patricia Urquieta y Sarah Botton, Agua y desigualdades urbanas (pp. 337-350). La Paz: CIDES-UMSA.

López Pérez, John Fredy, Dora Luz Delgado Gómez y Liliana Vinasco Torres (julio-diciembre, 2005). "La interfase urbano rural como territorio 
y espacio para la sostenibilidad ambiental". Revista Ingenierías Universidad de Medellín, 4(7), 29-41.

Los Tiempos (15 de febrero de 2018). "Desborde de río causa destrozos en Palca y alcalde pide ayuda". Los Tiempos [sección País]. Recuperado de https://www.lostiempos.com/actualidad/pais/20180215/desborde-rio-causa-destrozos-palca-alcalde-pide-ayuda

Modesto (15 de febrero de 2020). Entrevista individual a un comunario de Retamani. Entrevistador: Carla Andrea Cardona.

Peña Collazos, Wilmar (julio-diciembre de 2009). "La violencia simbólica como reproducción biopolítica del poder". Revista Latinoamericana de Bioética, 9(2), 62-75.

Perales Miranda, Víctor Hugo (agosto de 2018). "La crisis del agua en La Paz: cambios y racionamiento del agua". Temas Sociales, 43, 97-124. Recuperado de http://www.scielo.org.bo/scielo.php?script=sci_arttext\&pid=S0040-29152018000200005\&lng=es\&tlng=es.

PROAGRIS (2012). Plan de Uso del Suelo (PLUS): Macrodistritos Zongo y Hampaturi Municipio de La Paz, Bolivia. La Paz: The Nature Conservancy-Gobierno Autónomo Municipal de La Paz.

Sánchez, Macías Ramiro Gonzalo (2002). Estructura de la canasta familiar en Bolivia. [Tesis de licenciatura en Economía]. Facultad de Ciencias Económicas y Financieras, Universidad Mayor de San Andrés, La Paz.

Spedding, Alison, Gumercindo Flores Quispe y Nelson Aguilar López (2013). Chulumani: flor de clavel: transformaciones urbanas y rurales, 1998-2012. La Paz: PIEB.

Tassi, Nico y María Elena Canedo (2019). "Una pata en la chacra y una en el mercado": multiactividady reconfiguración rural en La Paz. La Paz: CIDES-UMSA. Torrico Foronda Escarley (2020). "Conflictos y tensiones por la propiedad del agua en el territorio de InterfaseUrbano-Rural de Hampaturi, municipio de La Paz". En Patricia Urquieta y Sarah Botton, Agua y desigualdades urbanas (pp. 337-350). La Paz: CIDES-UMSA.

Urquieta Crespo, Patricia (2019). "Los desafíos de la desigualdad urbana: agua y conflictos territoriales en el municipio de La Paz". En Alfredo Seoane y Luis Claros, Bolivia en el siglo XXI Transformaciones y desafios (pp. 277-294). La Paz: Plural editores. 